\title{
On the proper characterization of tooling motions and initial conditions in powder die compaction modeling
}

\author{
J.A. Hernández ${ }^{\mathrm{a}, \mathrm{b}, *}$, J.C. Cante $^{\mathrm{b}}$, J. Oliver ${ }^{\mathrm{c}}$, R. Weyler $^{\mathrm{b}}$ \\ a International Center for Numerical Methods in Engineering (CIMNE), C/Gran Capitán S/N, Campus Nord U.P.C., Edifici C-1, 08034 Barcelona, Spain \\ ${ }^{\mathrm{b}}$ E.T.S. Ingenierías Industrial y Aeronáutica de Terrassa, Technical University of Catalonia, C/Colom, 11,08222 Terrassa, Spain \\ ${ }^{\mathrm{c}}$ E.T.S. de Ingenieros de Caminos Canales y Puertos de Barcelona, Technical University of Catalonia, Campus Nord U.P.C., Edifici C-1, Jordi Girona 1-3, 08034 Barcelona, Spain
}

\section{A R T I C L E I N F O}

\section{Article history:}

Received 30 October 2010

Received in revised form 16 February 2011

Accepted 5 March 2011

Available online 15 March 2011

\section{Keywords:}

Powder metallurgy

Cold die compaction

Finite element modeling

Tooling motions

\begin{abstract}
A B S T R A C T
The present paper is concerned with the finite element modeling of Powder Metallurgy $(\mathrm{P} / \mathrm{M})$ cold die compaction process. Rather than on material constitutive theories or on numerical algorithmic issues, attention is confined exclusively on an scarcely addressed issue in the $\mathrm{P} / \mathrm{M}$ modeling literature: the proper characterization of the boundary (tooling motions) and initial conditions of the problem. A case study of the compaction of an axially symmetric multilevel adapter in an advanced CNC press machine is used to convey the relevance of the accurate representation of these input data in the quality of model predictions. It is shown that unawareness or deliberate simplification of apparently insignificant details in this respect may cause errors far overshadowing those introduced by deficiencies in either the constitutive model or in the corresponding algorithmic solution procedure. The discussion of this case study serves also to provide useful modeling guidelines; illustrate frequent difficulties, as the unavailability of some information when guessing starting conditions; and reveal subtle, yet relevant for modeling purposes, technical details of advanced CNC press machines.
\end{abstract}

(ㄷ) 2011 Elsevier B.V. All rights reserved.

\section{Introduction}

It would be difficult to overstate the potential beneficial impact that the systematic and routine application of computational modeling in the Powder Metallurgy ( $\mathrm{P} / \mathrm{M})$ industry - still heavily contingent upon trial-and-error procedures and rules-of-thumb based on prior experience - could have on the cost and efficiency of the whole $\mathrm{P} / \mathrm{M}$ manufacturing process. In the design of unconventional complex parts, for instance, the performance and reliability of a projected tooling could be assessed by means of computational simulations before the costly process of construction and mounting begins; scrutiny of density and/or cohesion distributions predicted by a computational model, on the other hand, could greatly assist in and expedite the diagnosis of the causes behind cracks observed in green compacts. The $\mathrm{P} / \mathrm{M}$ industry is largely aware of these potential benefits and, through collaborative efforts with research and academic entities-such as the thematic networks P/M Modnet (Federzoni et al., 1999) and P/M Dienet (Brewin and Federzoni, 2006), have encouraged in recent years the improvement of the "quality" of the existing computational models, specially, those employed in the simulation of cold die compaction processes.

\footnotetext{
* Corresponding author at: International Center for Numerical Methods in Engineering (CIMNE), C/Gran Capitán S/N, Campus Nord U.P.C., Edifici C-1, 08034 Barcelona, Spain. Tel.: +34 934017306.

E-mail address: jhortega@cimne.upc.edu (J.A. Hernández).
}

The notion "quality" in $\mathrm{P} / \mathrm{M}$ computational modeling can be interpreted in diverse ways; it depends on the goal, complexity and accuracy demands of the analysis. For a conventional, one-level part in which the $\mathrm{P} / \mathrm{M}$ designer can draw upon past experience, and in which dimensional tolerances are not an issue, using an elaborate finite element model with several hundred thousand degrees of freedom amounts to overkill; an elementary "column model" - one in which mass transfer is ignored - is more commensurate with the design needs in this case. In the present paper, however, it is presupposed that the analysis does have to meet stringent accuracy requirements; the notion "quality" will be thereby interpreted here as ability to reproduce, with fidelity, experimentally measured responses. Increasing the "quality" of the model will be regarded as tantamount to improving the agreement between measured and computed responses.

According to Delleur (2007), discrepancies between computed and experimentally measured responses are always the manifestation of errors, from the modeling side, of three markedly distinct type. Firstly, a mathematical model, however sophisticated, is a simplified picture of physical reality; errors, thus, will invariably arise because of both the deliberate (or unintentional) neglect of certain phenomena, on the one hand, and the inappropriate representation of many others, on the other hand. A second category includes those errors incurred in the equation-solving process (discretization, truncation, round-off and other numerical errors). Lastly, but by no means less significantly, uncertainties and inadequacies in the specification of the input data of the problem - 

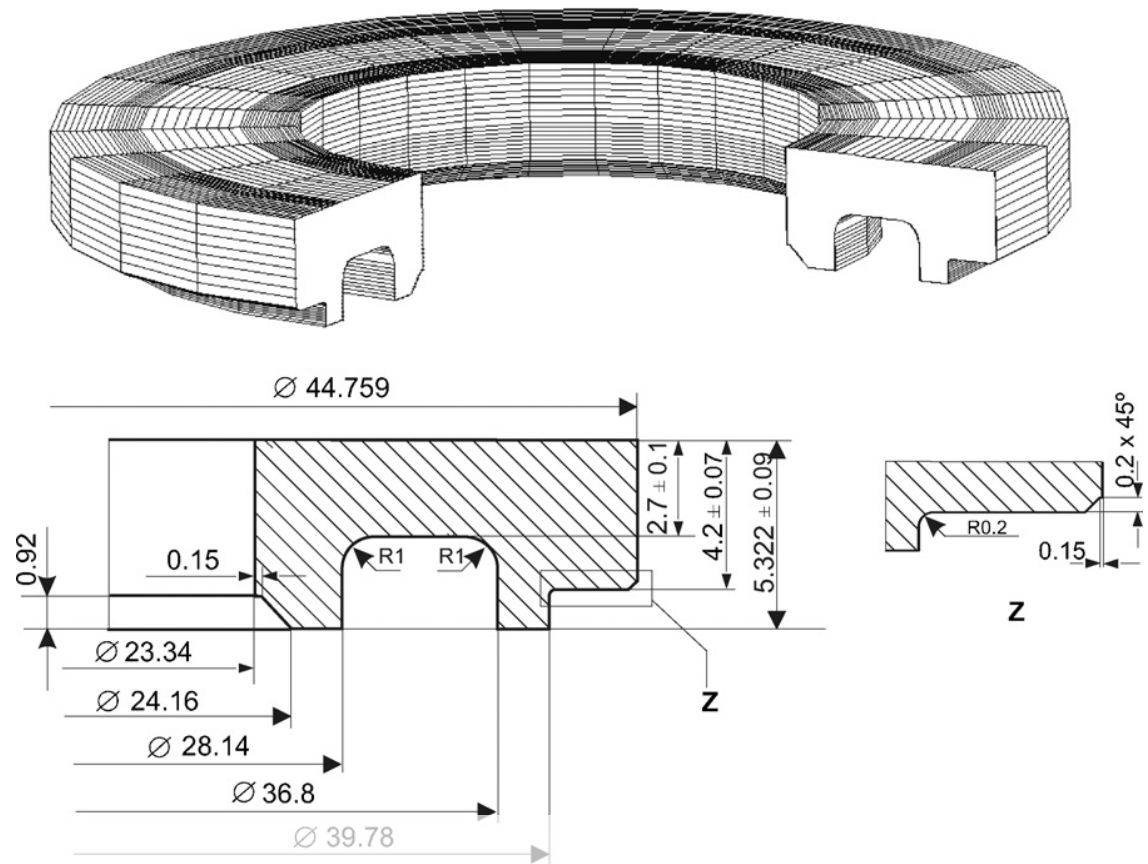

$\mathbf{Z}$

Fig. 1. Geometry of the part (dimensions in $\mathrm{mm}$ )

boundary and initial conditions - describing a particular situation can also contribute to differences between predicted and measured responses.

A survey of P/M modeling literature shows that, to date, model improvement efforts undertaken in the P/M research com have focused almost exclusively on reducing the first source of errors. Since the vast majority of existing models are based on the continuity assumption, basically to develop mo to describe and predict wit ior and powder-tooling relatively recent state- 6 models, see Biswas (2005). Strategies for reducing the second type account for subtle and often intricate features of press performance. Unawareness or deliberate oversimplifications of these details can cause errors in the computed response far overshadowing those introduced by flaws in either the constitutive model or the corresponding algorithmic solution procedure.

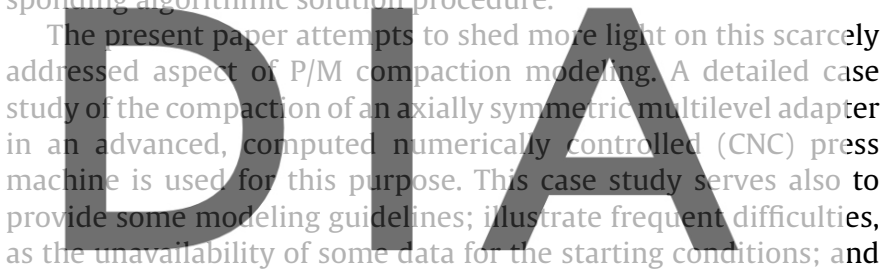
reveal relevant technical features that, although probably trivjah for rowder metallwy teghqugists may scane notice by researchers, especially lithey possess deflcient backglound knowledge of advanced CNC press machines. The authors have struggled to synthesize the experience and insight gained from this case study; collect and assimilate related recommendations scattered throughout $\mathrm{P} / \mathrm{M}$ literature; and present all this information in a manner that, hopefully, can aid and enlighten other users of $\mathrm{P} / \mathrm{M}$ modeling technology facing the task of defining the boundary and starting conditions of $\mathrm{P} / \mathrm{M}$ cold die compaction problems.

\section{Modeling of the compacting tool set}

The geometry and dimensions of the analyzed four-level part are displayed in Fig. 1. Note the relatively low height of the part: the finished lengths of the thinner and thicker levels of the part are only $2.7 \mathrm{~mm}$ and $5.32 \mathrm{~mm}$, respectively; this attribute will make changes in density induced by inaccurate definition of boundary conditions readily perceptible. For instance, were the part perfectly cylindrical, an error in describing the position of one of the tooling members of, say, $0.2 \mathrm{~mm}$, which is in the order of the value of the punches elastic deflection, would induce a totally unacceptable discrepancy in the predicted density of about $0.4 \mathrm{~g} / \mathrm{cm}^{3}$.

The word "model", in its broader sense, refers to the representation (mathematical in our context) of a physical system-the target system. In the case of $\mathrm{P} / \mathrm{M}$ cold die compaction, this system would demanding high accuracy, the modeling of the boundary conditions can become a complex issue in its own right, since one has to 


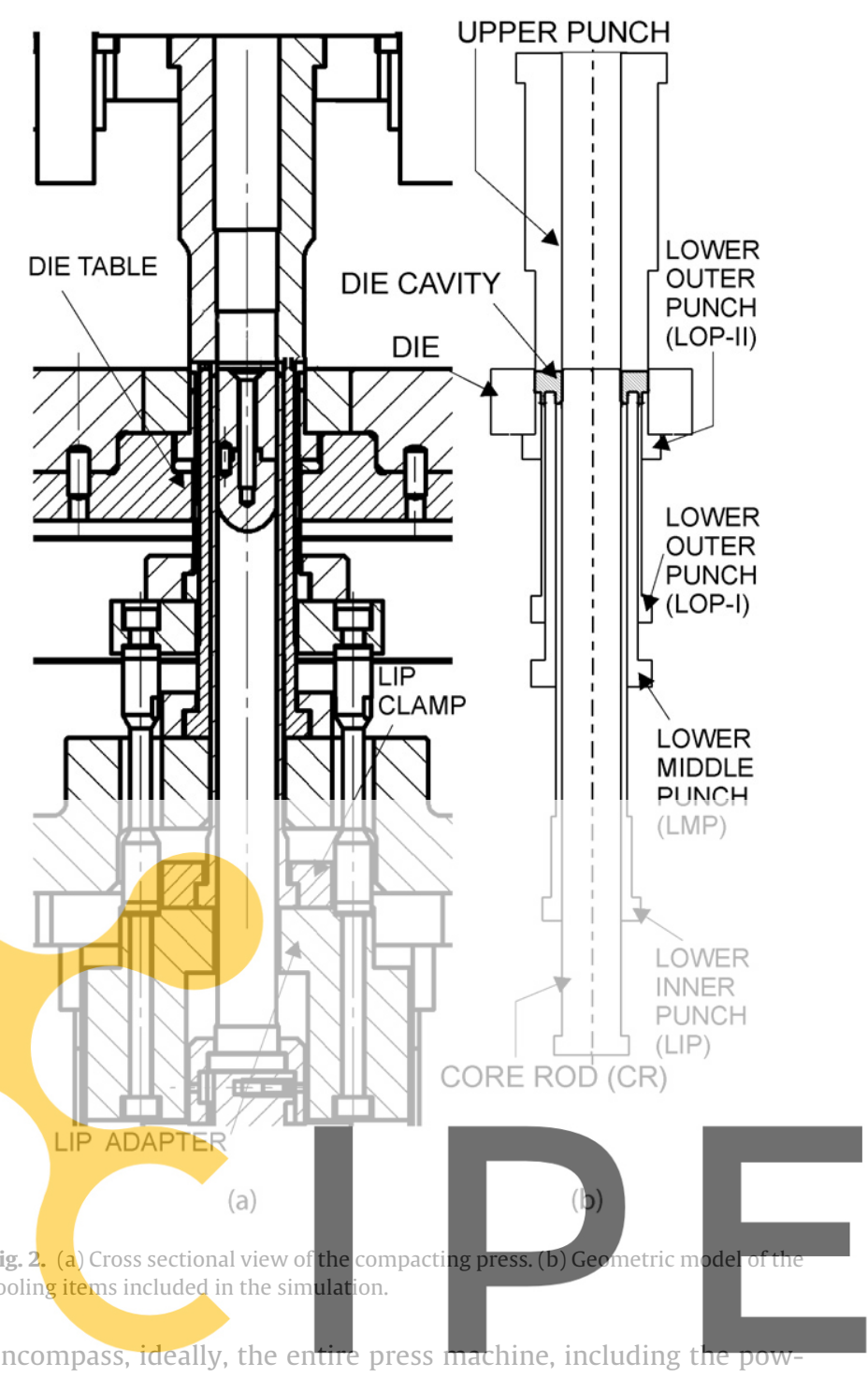

der contained in the die cavity, punches, core rod, die, press fittings,
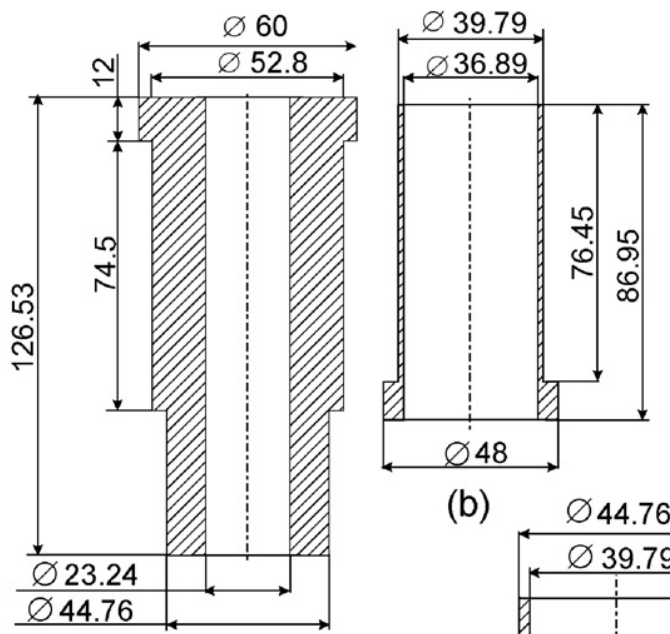

(b)

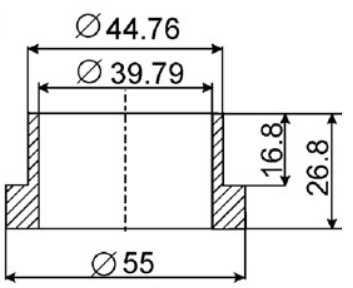

(c)

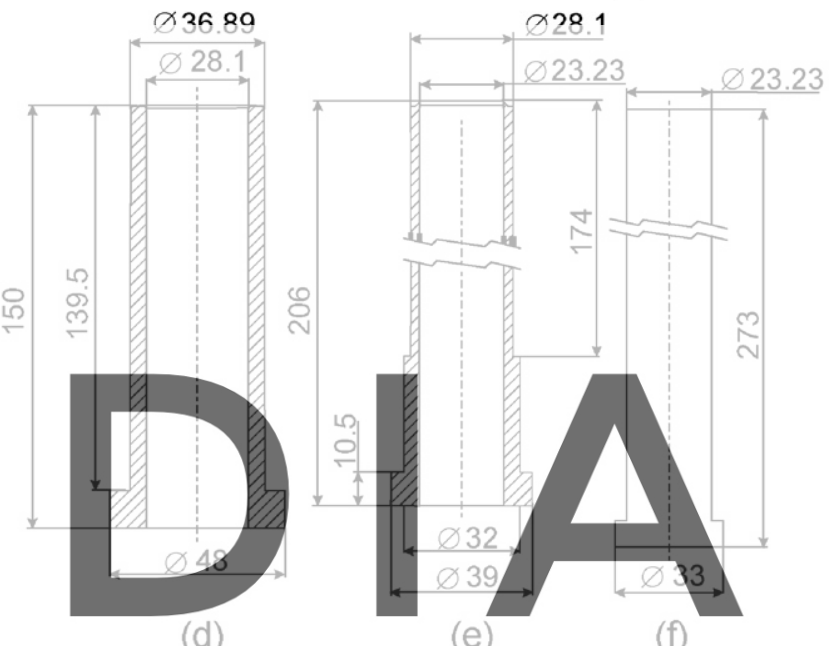

(e)

$(f)$

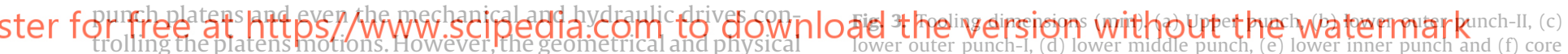

characterization of all these press members would imply a daunting labor and a dramatic increase in computational effort. It is usually more practical to include only the powder contained in the die and those press elements directly involved in forming the part, the socalled tool set: upper and lower punch(es), the die and the core rod. Punch platens, punch holders and punch adapters ca be excluded from the system on the basis of their larger rigidity, in comparison with punches. However, as it will emerge later (Section 3), these press elements do deflect under high pressures; the corresponding deflections should be eventually incorporated to the analysis as perturbations or deviations of the corresponding platen motion.

In Fig. 2a, the schematic of the multi-platen press machine employed in manufacturing the considered part is shown. The accompanying drawing, Fig. $2 b$, represents the tooling assembly included in the modeling, which comprises: an upper punch (UP); four lower punches (LIP, LMP, LOP-I and LOP-II); a die; and a core rod. Observe that the lower outer punch labeled as LOP-II is mounted on the die table, in a stepped die or shelf die fashion (Ferguson and Krauss, 1990). The dimensions of the core rod die and punches are shown in Fig. 3. In order to predict elastic deflections with the maximum level of accuracy, it is crucial to account for the actual length and the different diameters sections of punches. By contrast, features such as the blend fillets that reduce stress concentrations at change of section can be safely washed out, as the main concern is the study of the powder behavior. Other factors related to the tool set that can be also ignored are, among others: the magnitude of the clearances between moving tools; the wear resistance of tools; and busting and buckling phenomena. Such a range of details is important in its own right and should be studied separately.

\section{Boundary conditions}

The actions exerted on the target system - through its boundary - by press members not explicitly included in the system are generically termed "external actions". In a continuum setting, i.e. when both tools and powder are treated as continuous bodies, these external actions represent mathematically the boundary conditions of the governing balance equations. Depending on the circumstances and the type of press used for pressing the part, boundary conditions can be specified as either displacement or traction (pressure) conditions on the portion of punch surface in contact with the clamp rings that fasten punches to their corresponding adapters (see Fig. 2). Whereas the action of mechanical drives should be mod- 


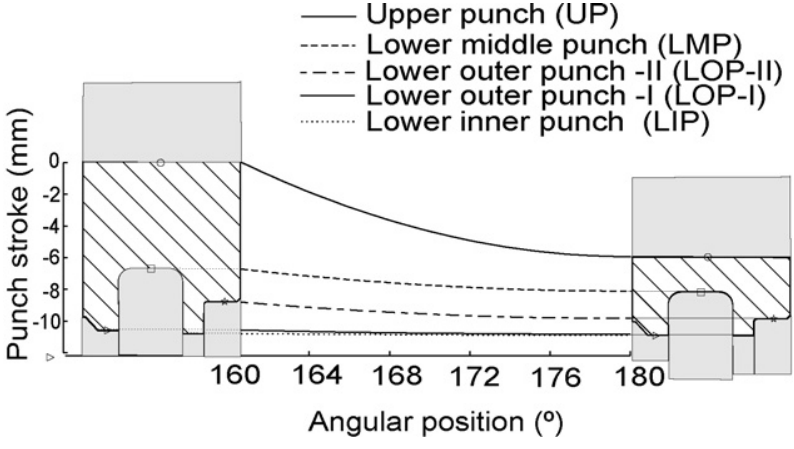

Fig. 4. Typical profile of tooling displacement during pressing.

elled always as prescribed displacement conditions, the effect of hydraulic drives can be represented either as prescribed displacements or by imposing traction conditions, depending of the type of press. In traditional hydraulic presses, for instance, the action of the punch drives has to be represented as traction conditions, for they can be programmed only to apply a fixed pressure. Advanced CNC press machines, on the other hand, are more versatile and hydraulically controlled punches can be both load-controlled and displacement-controlled. It is important to note that, in simulating the same pressing process, prescribed conditions can switch from traction type to displacement type, or vice versa. For example, a standard hydraulic press may be equipped with a mechanical stop to limit the downward ram movement, and therefore control the finished length of the corresponding level. When such mechanical stop comes into action, fixed pressure conditions should be instantaneously replaced by a zero displacement constraint.

The multi-platen CNC press used for pressing the studied part incorporates a combination of mechanically and hydraulically driven systems. The final col of an electric motor; a con of the main shaft of this upper ram. Consequent ing the pressing portion given by the following expl

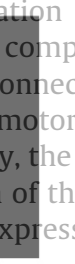

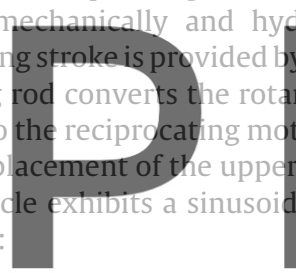

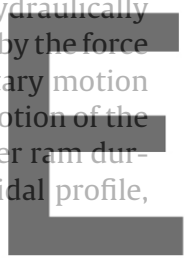

equation expresses such a condition:

$v_{p}=f_{p} v_{u p}$

where $v_{u p}$ is the linear velocity of the upper ram and $v_{p}$ represents the downward velocity of the corresponding lower ram. The condition of vanishing velocity at the bottom dead centre, $v_{u p}\left(\varphi=180^{\circ}\right)=0$, enables us to directly write:

$u_{p}=f_{p} u_{u p}$

i.e., given an upper ram pressing stroke $u_{u p}$, the desired stroke for the lower punches can be adjusted by changing the constant $f_{p}$. The proportionality parameter $f_{p}$, henceforth referred to as motion scale factor, must be less than one in order to ensure that pressing takes place; they constitute one of the basic operating parameters of the CNC press machine, that is, parameters that can be easily modified by the $\mathrm{CNC}$ operator by interacting with the $\mathrm{CNC}$ display screen.

\subsection{Theoretical versus true boundary conditions}

The displacement diagrams described in the preceding discussion, and illustrated in Fig. 4, are the motions that, theoretically, mechanical and hydraulic drives impart to their corresponding rams or platens. The easier, and most frequent, route when modeling compaction is to directly take these platen motions as the boundary conditions of the problem, that is, as the displacements prescribed at punch faces in contact with clamp rings. This simplification tendency often goes further, and complex displacement profiles are approximated by more elementary functions so as to facilitate their handling (sinusoidal curves replaced by straight lines, for instance).

These approximations are, to some extent, justifiable when the goal of the simulation is to merely elucidate qualitative features
of the process, but they are not when high predictive accuracy is
a must. The exact of true displacements of the supported portions
of punches, core rod and die seldom coincide with the correspond-
ing platen motions (henceforth termed "thedreticd" displacement, as
opposed to "true" displacements). In analyzing an already manu-
factured part, and provided that the CNC press has displacement
transducers located sufficiently close to the punch clamps, the "true" motion curves might be actually at the disposal of the P/M

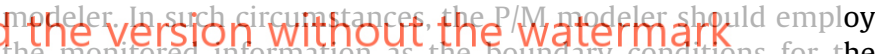
numerical simulation.

Unfortunately, very frequently, as when attacking a design problem or when testing numerically alternative pressing schedules, true displacement curves are not available; the analyst is faced in these cases with the task of having to anticipate or predict potential deviations from the theoretical tooling motions. Of invaluable help for this purpose is to, with the benefit of hindsight, examine and rationalize the discrepancies between theoretical and true motions observed either in compacting parts requiring similar compaction tonnage - in the case of design analysis - or in producing the same part but under different pressing and ejection schedules. The following classification can be also of great assistance in such a task; it synthesizes the most relevant deviations detected between true and theoretical displacements in the studied case.

(a) Deviations due to a poor characterization of the tooling subsystem. The fact that only punches, core rod and die are included in the model introduces an unavoidable error. Excluded press members, such as punch holders, punch adapters and platens also deflect under high pressures. However, the major contribution to this error can be attributed to the existence of adjustable mechanisms inserted between a punch and its corresponding platen. In the analyzed press machine, for instance, hydraulic devices to speed up or slow down to correct the velocity of the punch motion so as to keep the proportionality. The following 


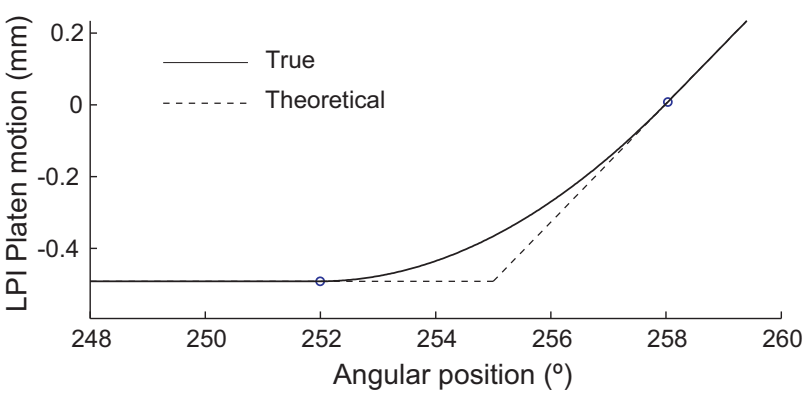

Fig. 5. Theoretical and true motion of the lower inner punch platen at the end of the ejection stage.

the force exerted by the hydraulic drives on the lower rams is transmitted to the lower punches through a practically rigid assembly of mechanically fastened elements; hence, little deviation is expected. By contrast, the hydraulically controlled mechanism inserted between the upper punch and the upper ram, alluded to earlier, contributes considerably to the discrepancy between theoretical and true upper punch motion because of the inevitably looseness (play) of the parts involved in such mechanism. To calibrate such error in full load operation, one can first measure the deviation in idle conditions, i.e., by compressing an empty die, and then consider that, approximately, the error increases linearly with increasing compacting pressure.

(b) Deviations due to overloading of lower rams. In CNC presses in which the stroke of lower punches is displacement-controlled during pressing, one cannot ignore the limited capacity of the hydraulic devices that governs their motions to sustain any applied force. If the programmed press kinematics induces an unduly high density, and consequently a high force, the servosystem controller may be pushed out of its regular operating conditions, and the lower ram will simply descend maintaining the level of pressure approxinnately constant, and thus not obeying the scheduled motion. Such a deviation can be included in the simulation by simply shifting the condition of presc displacement to prescribed traction on the affected low cribed when the corresponding force threshold is exceeded. Later on, the impact of overlooking such limited capacity will be assessed

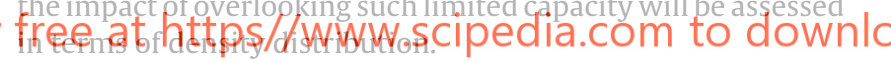

(c) Deviations due to interpolation of punch motions. As can be checked in Fig. 5, the actual position of the corresponding platens is slightly altered in the blended portions of the displacement diagrams. These deviations may be of the order of magnitude of the elastic deflection of the longer punches; hence their effect should be included when studying the ejection process. Furthermore, tooling displacement diagrams exhibiting sharp corners are physically unacceptable, since they imply instantaneous change of velocity and therefore infinite accelerations. In practice, velocity is ramped up or down gradually so as to avoid these unrealistic accelerations. These details are normally ignored in designing - and also very frequently in modeling - the compaction process, mainly due to obvious simplicity reasons. However, from the modeling standpoint, one cannot underestimate the benefits arising from using the actual smooth displacement profiles instead of the sharp ones: computability is affected adversely by rough input data (Belytschko and Mish, 2001).

Not every difference observed between "true" and "theoretical" curves is amenable to theoretical predictions. Some deviations may display an - apparently - random pattern, and hence they could not be predicted on the basis of a deterministic analysis;

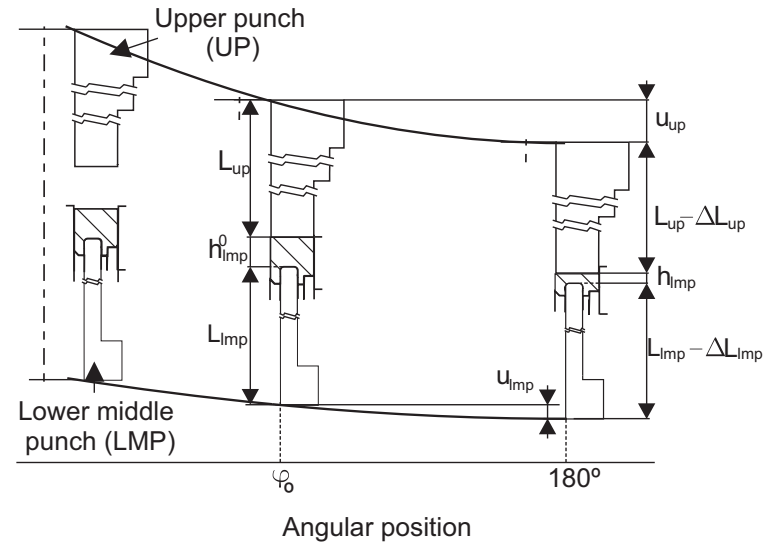

Fig. 6. Pressing sequence, indicating the motion of the upper punch and the lower middle punch. The angle $\varphi_{\mathrm{o}}$ denotes the point of the cycle at which the upper punch enters the die cavity. The pressing stroke ends at $\varphi=180^{\circ}$ (bottom dead centre).

only through statistical analysis the assessment of the impact of the individual and combined effect of such deviations would be possible. Needless to say, the borderline between what is predictable or unpredictable depends largely on the beholder and the degree of knowledge on the press machine. As experience accumulates, initially presumed unpredictable discrepancies can be converted into predictable ones. Conversely, presumably foreseeable deviations turning into uncontrolled perturbations may indicate either malfunction of any of the press machine elements or poor maintenance conditions.

\section{Estimation of initial conditions}

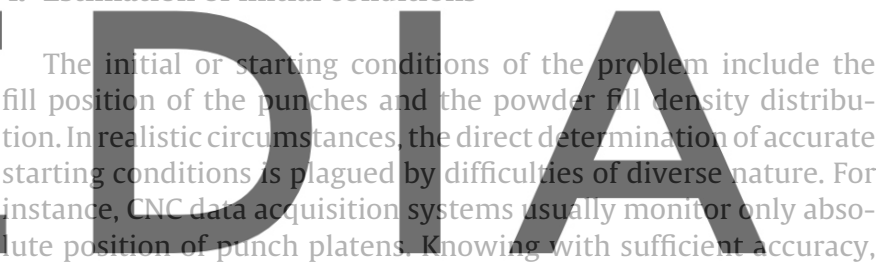
say $0.1 \mathrm{~mm}$, the position of the bottom face of lower punches would require thus careful measurements of the length of the assembly

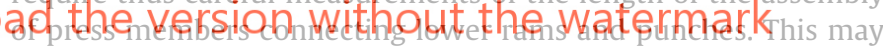
be somewhat difficult, and impractical, to achieve if, for example, the die set has been removed and disassembled for repairing when tackling the computer analysis.

In the ensuing discussion, the procedure followed here to confront the unavailability of reliable information concerning the aforementioned starting conditions will be outlined. Note that the unavailability of such data creates a scenario very similar to that encountered in the analysis of a trial design, in which one has to guess appropriate initial die cavity dimensions consistent with the finished lengths of each of the levels within the compacted part; hence, the procedure described in the sequel is nothing but the inverse analysis typically used in $\mathrm{P} / \mathrm{M}$ designing. The peculiarity that renders this inverse analysis worthy of special consideration here arises from the combination of two facts: tool motions are nonlinear functions of the angular position (time); and punch elastic deflections have to be included in the calculation of the die cavity dimensions.

The fundamental relation between the prescribed displacement on a given punch and the height of the level formed by this punch is illustrated in Fig. 6 for the particular case of the lower middle punch (LMP). This relation can be expressed analytically as

$h_{l m p}^{0}-h_{l m p}=u_{u p}\left(\varphi_{0}\right)-u_{l m p}\left(\varphi_{0}\right)-\left(\Delta L_{u p}+\Delta L_{l m p}\right)$ 


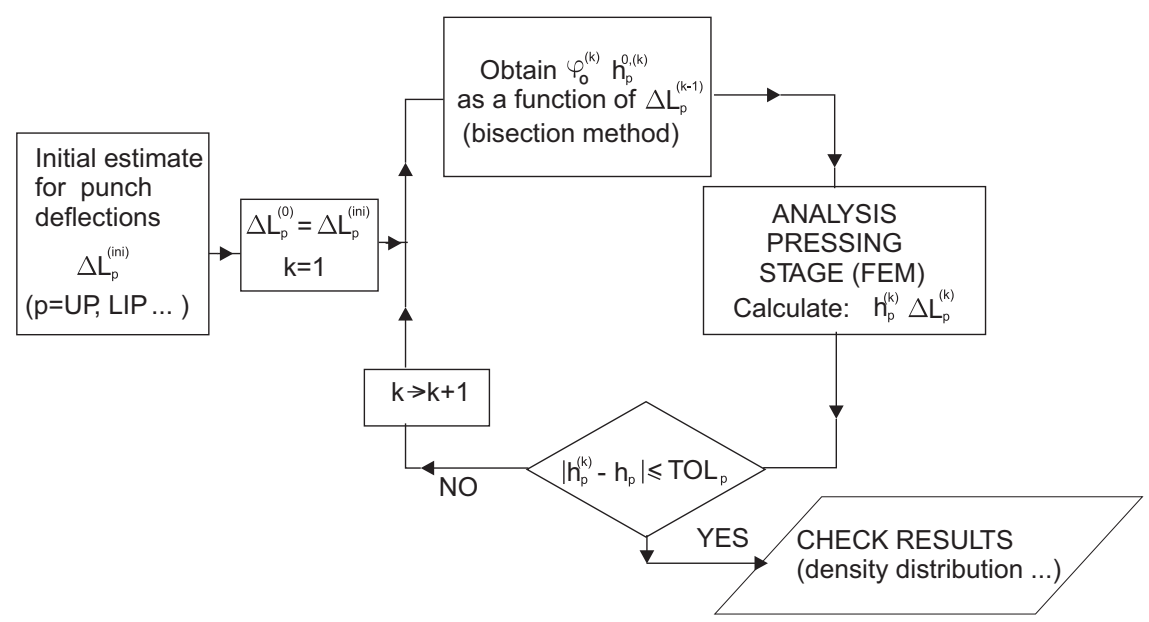

Fig. 7. Flowchart indicating the computational cycle used for estimating the initial die cavity dimensions.

The meaning of each term of the above equation is also clarified in Fig. 6. The quantity $h_{l m p}$ is the length, measured at the end of the pressing stage, of the level formed by the lower middle punch. This length is approximately the length measured upon ejection from the die, except for a small correction due to springback. The depth of fill or fill height corresponding to the thickness level formed by the LMP is denoted by $h_{l m p}^{0}$, and it is defined as the distance from the working end of the upper punch to the working end of the lower middle punch when pressing commences. The displacements prescribed at the top and bottom surfaces of upper and lower middle punches are symbolized by $u_{u p}$ and $u_{u p}$, respectively. As already discussed, the shape of the displacement profiles are, theoretically, sinusoidal (see Eqs. (1) and (3)), although they may be eventually affected by some deviations. Finally, the terms $\Delta L_{u p}$ and $\Delta L_{\text {lmp }}$ stand for the total elastic deflections in the axial
direction experienced by the upper punch and the lower middle
punch, respectively. Similar equations to Eq. ( 4$)$ dan be derived for
the other three thickness levels:
$h_{\text {lip }}^{0}-h_{\text {lip }}=u_{\text {up }}\left(\varphi_{0}\right)-u_{\text {lip }}\left(\varphi_{0}\right)-\left(\Delta L_{\text {up }}+\Delta L_{\text {lip }}\right)$ $h_{\text {lop }}^{0}-h_{\text {lop }}=u_{\text {up }}\left(\varphi_{0}\right)-u_{\text {lip }}\left(\varphi_{0}\right)-\left(\Delta L_{\text {up }}+\Delta L_{\text {lop }}\right)$ the initial angular position. The closure for this system, as may be surmised, is provided by the conservation of mass equation:

$\rho_{0} V_{0}\left(h_{\text {lip }}^{0}, h_{l m p}^{0}, h_{l o p^{\prime}}^{0}, h_{l o p^{\prime \prime}}^{0}\right)=\rho_{f} V_{f}\left(h_{l i p}, h_{l m p}, h_{l o p^{\prime}}, h_{l o p^{\prime \prime}}\right)$

wherein $\rho_{0}$ and $\rho_{f}$ are the (average) initial and final density, respectively, $V_{0}$ stands for the volume occupied by the powder at the onset of pressing and $V_{0}$ denotes the volume of the finished part. Absent reliable experimental measures of the average initial density $\rho_{0}$, this variable can be taken as the apparent density of the powder. ${ }^{1}$

To obtain the solution of the resulting system of equations, the values of the unknown quantities $h^{0}$ have to expressed as a function of $h$ and $u\left(\varphi_{0}\right)$. Upon substitution of $h^{0}$ in Eq. (9), the system is reduced to a single equation in the unknown $\varphi_{0}$. Due to its non-linear character, this equation is not amenable to analytical solution and, consequently, recourse to approximate algorithms, as the bisection method, is to be made/Once the angular position $\varphi_{0}$ has been obtained, the dimensions of the hitial cavity $h^{0}$ can be retrieved from Eqs. (5) to (7); with these estimated initial conditions at one's disposal, one has all the basic ingredients to construct the geometric madel, impose boundary conditions and, finally, undertake the finite element analysis of the pressing stage. However, it is by no means guaranteed that the final lengths
Expressions (4) to (7) form a system of four equations - one equation for each lower punch - with ten unknowns: the fill heights corresponding to each level (4 unknowns); the elastic deflections of punches ( 5 unknowns); and the angular position at which the upper punch comes into contact with the powder ( 1 unknown), denoted as $\varphi_{0}$. The elastic deflections can be estimated either on the basis of available data for similar parts, or by means of the uniaxial approximation:

$\Delta L=\frac{\sigma_{z}\left(\rho_{f}\right)}{E_{\text {tool }}} \sum_{k=1}^{n_{r}} \frac{R_{1}^{2}-R_{i}^{2}}{R_{k}^{2}-R_{i}^{2}} L$

The above equation follows from assuming that a uniaxial stress state prevails throughout the tubular punch. The constant $E_{\text {tool }}$ is the Young's modulus characterizing the tooling material whereas $R i$ stands for the inner radius of the corresponding tubular punch and $R k(k=1,2 \ldots)$ denotes the outer radii of each cross section of the punch, sorted by increasing magnitude. The magnitude of the axial pressure $\sigma_{Z}$ acting on the punch face can be estimated from the compressibility curve as the axial pressure corresponding to the final density $\rho_{f}$ of the compact. Substituting these estimations for the elastic deflection of punches in Eqs. (4) to (7) leads to a system of four equations with five unknowns, namely the 4 fill heights and

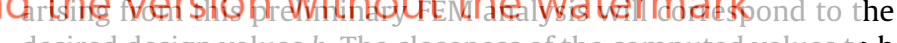
desired design values $h$. The closeness of the computed values to $h$ relies on the quality of the deflection estimations: a poor estimation of $\Delta L$ will invariably lead to incorrect final dimensions. In the case at hand, the relatively small thickness of the part $(2.7 \mathrm{~mm}$ in its thinner lever) aggravates the situation, since such inaccuracies in estimating $\Delta L$ will translate in inaccurate density predictions.

A strategy that proves efficient in successively improving the quality of these estimations is to use the punch deflections computed in the FE analysis as the estimations for a subsequent inverse analysis. This iterative strategy is schematically described in the flowchart displayed in Fig. 7. Iterations are halted when the difference between computed finished lengths and design values $h$ are within prescribed tolerances. In our case, such tolerances are taken as the dimensional tolerances contained in the customer's specification (see Fig. 1). A more in-depth appreciation of this procedure will be gained through the analysis presented in the following.

\footnotetext{
1 For intricately shaped parts of considerable height, fill density distributions are often far from uniform; thus, results obtained on the assumption that the average initial density coincides with the apparent density might be not correct. This fact should be taken also into consideration in explaining, for such parts, discrepancies between computed and experimental final densities; see Coube et al., 2005; Federzoni et al., 1999; and references therein for further details in this respect.
} 


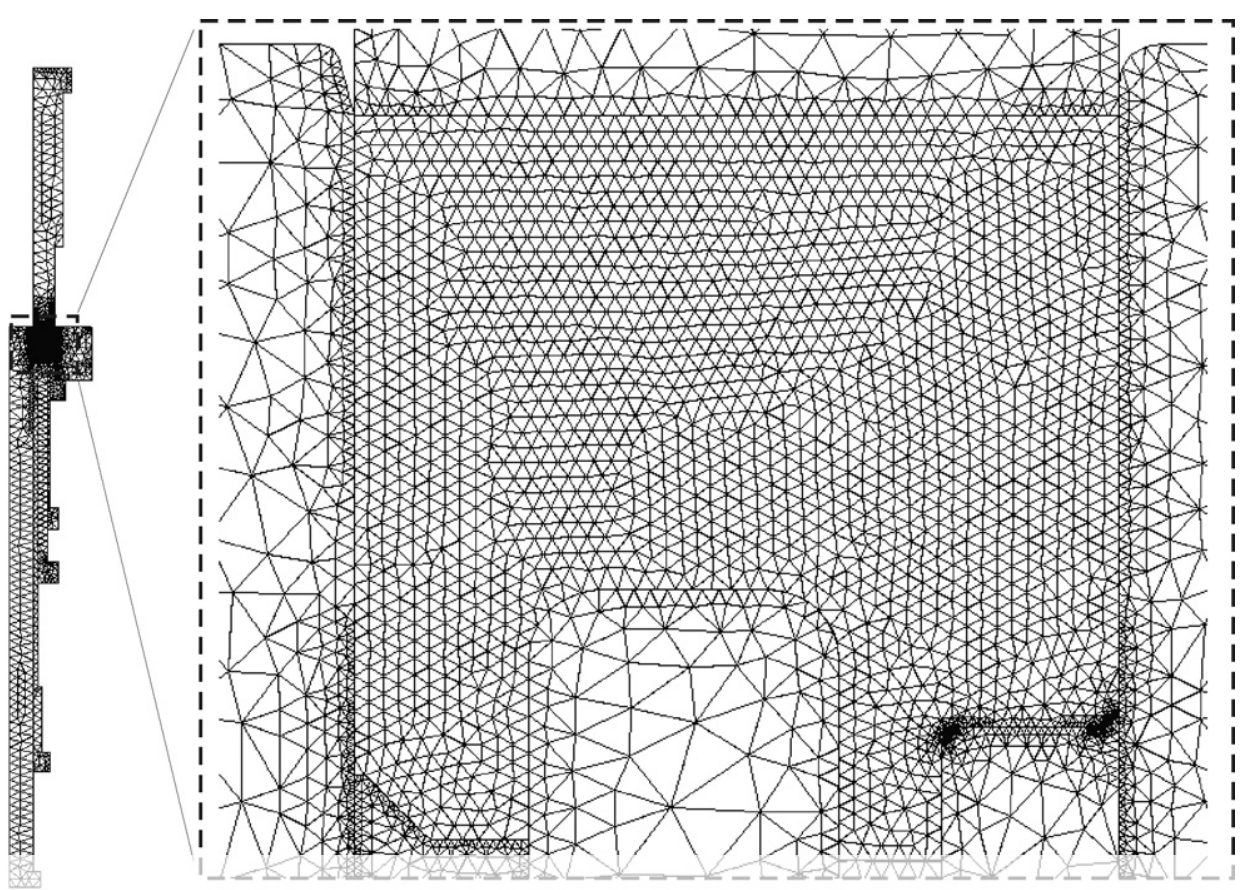

(b)

5. Assessment of the effect of an inaccurate description of tooling motions
Table 1

Motion scale factors
In this section, the comp butions produced by using prescribed conditions on too conditions closer to true tool
be examined and compared looking some of the deviatic powder employed in making t. powder with apparent density $\rho_{a p p}=3.25 \mathrm{~g} / \mathrm{cm}^{3}$. Fill density, which is assumed uniform throughout the die cavity, is taken as this

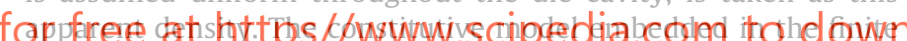
element code used for the calculations is the large strains, elastoplastic Drucker-Prager cap type model proposed by the authors in Heinández et al. (2011); calibrated model parameters for the considered Distaloy AE can be also found in this reference.

Friction between the powder mass and the faces of the toolsdie walls and core rod is modelled via a friction Coulomb law, with coefficient $\mu=0.12$. The elastic behavior of the tooling, on the other hand, is characterized by a Young's Modulus $E_{\text {tool }}=210 \mathrm{MPa}$ and a Poisson's ratio $v_{T O O L}=0.3$. The axial symmetry of the part is exploited and the study is concentrated on a characteristic radial section so that the FE analysis can be accomplished in two dimensions. The initial finite element mesh is shown in Fig. 8. The average size of the elements of the powder body is $l_{e}=0.25 \mathrm{~mm}$; the size of the elements at the boundaries of punches and die in contact with

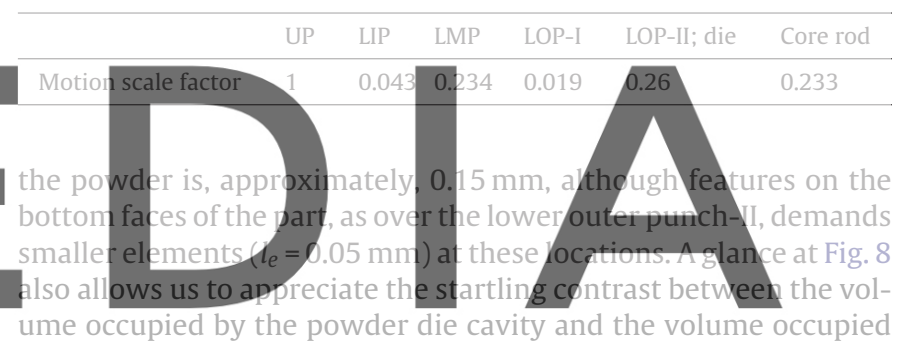
by the tooling set. oad the versior

5.1. Results using theoretical punch displacements

The theoretical or nominal displacement profile for the upper punch reciprocating motion can be obtained from Eq. (1). The values of the lengths of the crankshaft and the connecting rod appearing in such equation are $l_{c}=90 \mathrm{~mm}$ and $l_{r}=580 \mathrm{~mm}$, respectively. On the other hand, the theoretical motions of lower punches, core rod and die are fully determined by the motion scale factors shown in Table 1.

The motion scale factor of the lower outer punch LOP-II and the die are identical, as they are mounted in the same platen. On the other hand, it follows from the motion scale factors of the LIP and LOP-I that those punches are held practically stationary during the pressing stage. The information tabulated in Table 2 serves to

Table 2

Iterative procedure for calculating the initial die cavity dimensions. Theoretical tooling motion case.

\begin{tabular}{|c|c|c|c|c|c|c|c|}
\hline & & & UP & LIP & LMP & LOP-I & LOP-II \\
\hline \multicolumn{3}{|c|}{ Tolerance in $h(\mathrm{~mm})$} & - & 0.09 & 0.10 & 0.09 & 0.07 \\
\hline$k=0$ & $\Delta L$ (Uniax. est) & & 0.307 & 0.352 & 0.245 & 0.130 & 0.036 \\
\hline \multirow{2}{*}{$k=1$} & $\varphi_{0}=160.2^{\underline{0}}$ & $\Delta L^{(k)}$ & 0.175 & 0.298 & 0.123 & 0.153 & 0.0241 \\
\hline & & $h^{(k)}-h$ & - & -0.162 & -0.080 & -0.080 & -0.068 \\
\hline \multirow[t]{2}{*}{$k=2$} & $\varphi_{0}=160.9^{\underline{o}}$ & $\Delta L^{(k)}$ & 0.195 & 0.197 & 0.167 & 0.0928 & 0.0204 \\
\hline & & $h^{(k)}-h$ & - & -0.070 & 0.055 & -0.049 & 0.003 \\
\hline
\end{tabular}




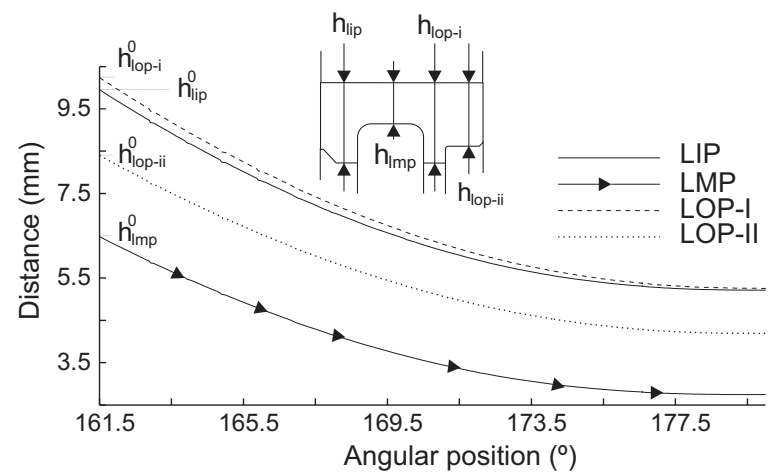

Fig. 9. Distance between working ends of upper and lower punches as a function of the angular position during the pressing cycle. Theoretical tooling motion case.

\section{FEM}

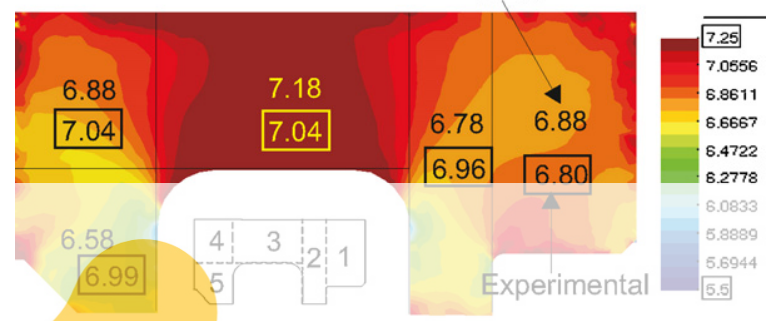

Fig. 10. Contour plot of density $\left(\mathrm{g} / \mathrm{cm}^{3}\right)$ computed at the end of the pressing stage. Theoretical tooling motion case.

illustrate the previousl of the initial die cavity dime deflections $\Delta L$ are obtainec The iterative sequence the computed finished lengt is within prescribed toler gence tolerance for the ower inne punch is not met, due to a too high initial estimation of its deflection. An additional computer run was hence required to achieve consistent initial conditions.

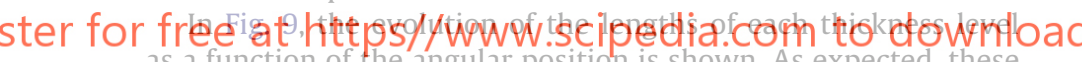
as a function of the angular position is shown. As expected, these curves mimic the sinusoidal pattern of the reciprocating motion of the upper ram. The contour plot of the density computed by the finite element method at the end of the pressing stage is displayed in Fig. 10. The level formed by the lower middle punch exhibits the highest density $\left(7.18 \mathrm{~g} / \mathrm{cm}^{3}\right)$, whereas the region with the lowest density $\left(6.80 \mathrm{~g} / \mathrm{cm}^{3}\right)$ is located above the lower inner punch. To enable direct comparison with experimentally measure densities, the part is divided into five volumes; the averaged density over those volumes is shown also in Fig. 10. Experimental and computed results are presented in Table 3. It is apparent that numerical results barely resemble the experimental density measures. Discrepancies between numerical and experimental data are especially unacceptable in region labeled as "five", just over the lower inner punch.

\section{Table 3}

Comparison between computed densities $\left(\mathrm{g} / \mathrm{cm}^{3}\right)$ using theoretical tooling motion $\left(\rho_{\text {num }}\right)$ and experimentally measured values $\left(\rho_{\text {exp }}\right)$.

\begin{tabular}{llrlrrr}
\hline Zone & 1 & \multicolumn{1}{l}{2} & \multicolumn{1}{l}{3} & \multicolumn{1}{l}{4} & \multicolumn{1}{l}{5} & Overall \\
\hline$\rho_{\text {num }}$ & 6.88 & 6.78 & 7.18 & 6.88 & 6.58 & 6.91 \\
$\rho_{\text {exp }}$ & 6.80 & 6.96 & 7.04 & 7.04 & 6.99 & 6.94 \\
$\rho_{\text {num }}-\rho_{\text {exp }}$ & 0.08 & -0.18 & 0.14 & -0.16 & -0.41 & -0.03 \\
\hline
\end{tabular}

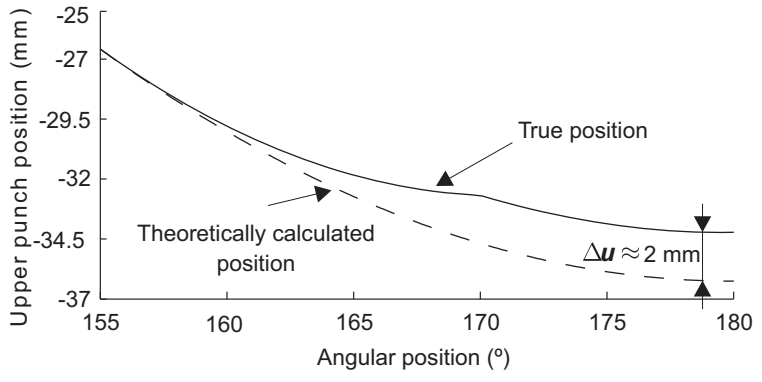

Fig. 11. Position of the upper punch ram. Theoretically calculated value (dashed line) and value monitored and recorded by the CNC data acquisition system (solid line).

\subsection{Results using "true" punch displacements}

The simulation of the pressing stage using prescribed conditions closer to the tool motions monitored by the CNC computer during the compression is discussed in the following. The discrepancies between the theoretically predicted and true motions of LIP, LOP-I, die and core rod are insignificant and the prescribed displacements are therefore the same as in the previous case. Bv contrast, substantial errors are detected in the description of the upper punch and lower middle punch motions. Fig. 11 shows the theoretical and "true" positions of the top face of the upper punch during the pressing portion of the cycle. The position recorded by the CNC data acquisition system exhibits a gradual deviation from the theoretically calculated reciprocating motion of the main shaft. At the bottom dead centre, the amplitude of this deviation can be estimated at approximately $2 \mathrm{~mm}$. This deviation is attributable to the looseness of the parts comprising the hydraulically operated mechanism that controls the force exerted by the upper punch during
ejection, which is located between the upper purch and the upper ram. In ad dition, in discussing the classification of what has been here terned "predictable deviations" (see Section 3.1), it was pointed out that the hydranlic drives operating the lower rams execute the scheduled motion only if the resulting force on each punch is below a certain threshold. In the case of the lower middle punch, the corresponding hydraulic servo-system operates under regular con-

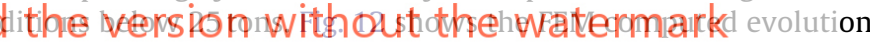
of the force exerted by the compacting powder on the lower middle punch using theoretical tooling kinematics, in which the stroke of the lower punches is assumed to be displacement-controlied during the entire pressing stage.

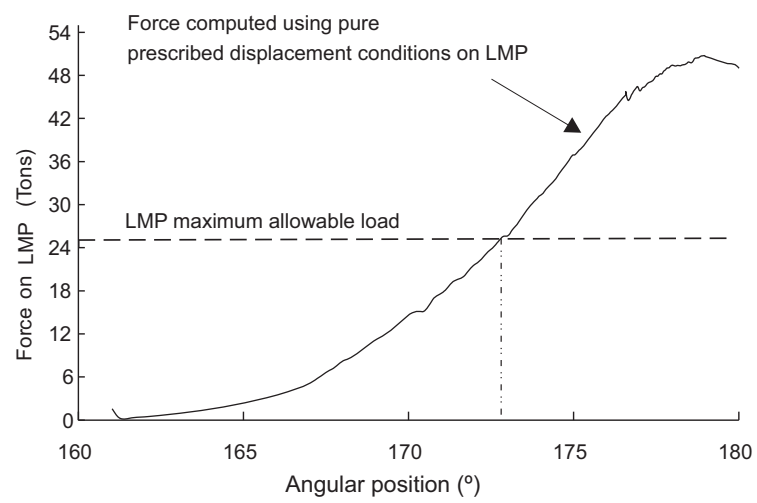

Fig. 12. Force on the lower inner punch computed using pure prescribed displacement condition on the lower middle punch. The horizontal dashed line indicates the threshold below which the hydraulic device controlling the LMP platen operates correctly. 
Table 4

Iterative procedure for calculating the initial die cavity dimensions. True tooling motion case.

\begin{tabular}{|c|c|c|c|c|c|c|c|}
\hline & & & UP & LIP & LMP & LOP-I & LOP-II \\
\hline \multicolumn{3}{|c|}{ Tolerance in $h(\mathrm{~mm})$} & - & 0.09 & 0.10 & 0.09 & 0.07 \\
\hline$k=0$ & $\Delta L$ (Previous simulat.) & & 0.175 & 0.298 & 0.123 & 0.153 & 0.0241 \\
\hline \multirow[t]{2}{*}{$k=1$} & $\varphi_{0}=156.7^{\circ}$ & $\Delta L^{(k)}$ & 0.182 & 0.325 & 0.136 & 0.196 & 0.016 \\
\hline & & $h^{(k)}-h$ & - & -0.0043 & -0.057 & 0.0125 & -0.034 \\
\hline
\end{tabular}

Table 5

Fill heights ( $\mathrm{mm}$ ) corresponding to each thickness level. Theoretical and "true" tooling motion cases.

\begin{tabular}{lrrrr}
\hline & \multicolumn{1}{c}{ LIP } & \multicolumn{1}{c}{ LMP } & LOP-I & LOP-II \\
\hline$h^{0}$ Computed using nominal displac & 10.22 & 6.70 & 10.52 & 8.63 \\
$h^{0}$ Computed using true displac & 10.87 & 6.47 & 11.23 & 8.20 \\
Difference (\%) & 5.97 & -3.54 & 6.37 & -5.35 \\
\hline
\end{tabular}

The information displayed in Fig. 12, however, makes apparent the inadequacy of such assumption: the computed force at the end of the pressing operation exceeds the maximum allowable force by a factor of almost two. This unduly high force on the LMP is intimately connected with the over-densification observed in the region over the lower middle punch (see Fig. 10). According to the suggestion given in Section 3, the prescribed condition on the lower middle punch must be modified to accommodate this limited capacity of the hydraulic device. For forces below 25 tons, displacements given by the expression $u_{\operatorname{lmp}}=f_{\operatorname{lmp}} u_{u p}$ will be imposed on the bottom surface of the punch; when the force rises slightly above 25 tons, the displacement condition will be replaced by a pressuretype condition so as to ensure that the lower middle punch moves downward maintaining the force in 25 tons.

The simulation of the pressing stage is carried out again with these new boundary conditions on both upper and lower middle punches. First, the starting illustrated in the flowchart tion of punch deflections en from the first iteration in theoretical motion case) ing such a procedure is set fort

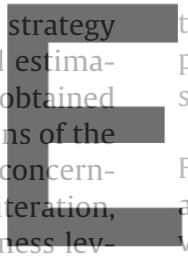
els and the design values are between the prescribed tolerances;
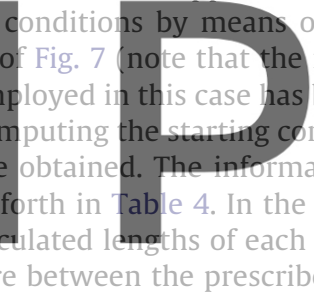

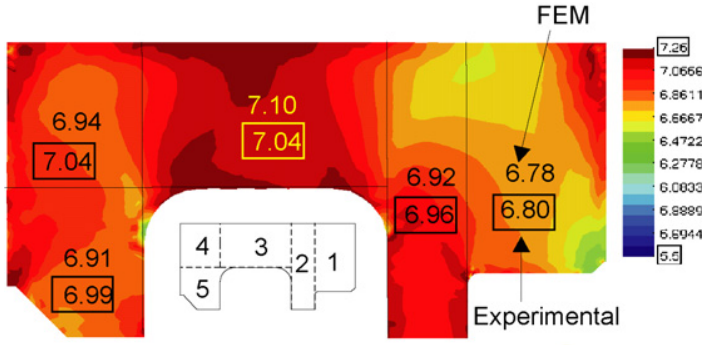

Fig. 14. Contour plot of density computed at the end of the pressing stage. "True" tooling motion case.

Table 6

Comparison of computed densities $\left(\mathrm{g} / \mathrm{cm}^{3}\right)$ using true $\left(\rho^{T R U E}\right)$ and theoretical $\left(\rho_{\text {num }}^{\text {THEOR. }}\right)$ tooling motion with experimentally measured values $\left(\rho_{\text {exp }}\right)$.

\begin{tabular}{|c|c|c|c|c|c|c|}
\hline Zone & 1 & 2 & 3 & 4 & 5 & Overall \\
\hline$\rho_{\text {num }}^{T R U E}$ & 6.78 & 6.92 & 7.10 & 6.94 & 6.91 & 6.93 \\
\hline$\rho_{\text {nUEm }}^{\text {THEOR. }}$ & 6.88 & 6.78 & 7.18 & 6.88 & 6.58 & 6.91 \\
\hline$\rho_{\exp }$ & 6.80 & 6.96 & 7.04 & 7.04 & 6.99 & 6.94 \\
\hline$\rho_{\text {num }}^{T R U E}-\rho_{\exp }$ & -0.02 & -0.04 & 0.06 & -0.10 & -0.08 & -0.01 \\
\hline$\rho_{\text {num }}^{\text {THEOR. }}-\rho_{\exp }$ & 0.08 & -0.18 & 0.14 & -0.16 & -0.41 & -0.03 \\
\hline
\end{tabular}

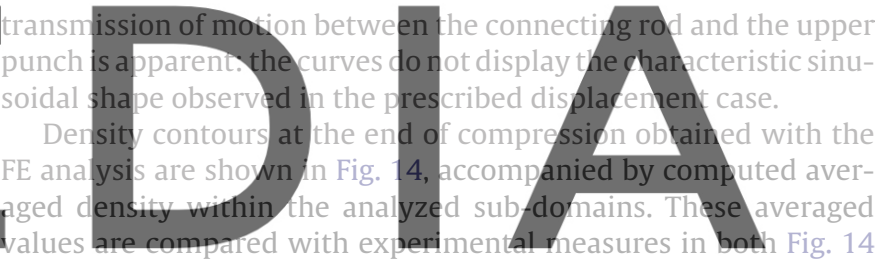
and Table 6.

Clearly numerically predicted densities obtained with the

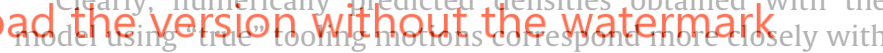
empirically measured densities than in the theoretical tooling motion case. Improvement is especially drastic in region 5, just over the lower inner punch, and more moderate in the other subdomains. Discrepancies between experiment and computations still persist - the maximum being $0.1 \mathrm{~g} / \mathrm{cm}^{3}$ - but are, according to a commonly held criteria in P/M modeling (see Modnet, 1999), within acceptable engineering accuracy. It can be concluded thus that the "quality" of both the constitutive equations that lie at the core of the model and the pertinent equation-solving algorithm - not discussed here, but in Hernández et al. (2011) and Hernández (2009), respectively - are satisfactory from a practical point of view: it was the deficient quality of what has been here termed "theoretical" input data that bears the blame for poor results.

\section{Conclusions}

The goal of the present work was to address an issue scarcely considered in the of $\mathrm{P} / \mathrm{M}$ literature: the accurate characterization of the boundary - tooling displacements and forces - and initial conditions in the modeling of die compaction processes. A detailed case study of the compaction of an axially symmetric multilevel adapter in an advanced CNC press machine has been used to convincingly show that the introduction of apparently
Fig. 13. Distance between working ends of upper and lower punches as a function of the angular position in the pressing cycle. "True" tooling motion case. 
innocuous simplifications, or the unintentionally neglect of some details in specifying the boundary conditions can cause substantial disagreement between computed and experimental results. It is, therefore, of paramount importance that one be properly versed in CNC press performance features so as to be aware of potential sources of deviations between "theoretical" and "true" tooling motions. Unawareness of such deviations may promote the tendency of rationalizing discrepancies as being due to flaws either in the constitutive model or in the corresponding algorithmic procedure; one would be tempted in these cases to either refine the constitutive equations or to increase the accuracy of the numerical approximation. But one does not increase the strength of a chain by improving the strong links: further refinements of these issues would be futile if the other aspect of the model contributing to the discrepancy between prediction and measurement - the input data - are not equally improved.

\section{Acknowledgements}

The Spanish Ministry of Science and Innovation, and the Catalan Government Research Department are gratefully acknowledged for financial support under grants BIA2008-00411 and 2009 SGR 1510, respectively. The authors wish to thank also AMES S.A. for supporting the experimental part of this research; a special debt of gratitude is owed to Enric Sánchez and Josep Lluis Celeiro for their endless patience in helping us navigate the intricacies of $\mathrm{CNC}$ press machines.

\section{References}

Belytschko, T., Mish, K., 2001. Computability in non-linear solid mechanics. International Journal for Numerical Methods in Engineering 52, 3-21.

Biswas, K., 2005. Comparison of various plasticity models for metal powder compaction processes. Journal of Materials Processing Technology 166, 107-115.

Brewin, P., Federzoni, L., 2006. Dienet: conclusions and achievements. Powder Metallurgy 49, 8-10.

Brewin, P.R., Coube, O., Doremus, P., Tweed, J.H., 2007. Modelling of Powder Die Compaction. Springer Verlag.

Coube, O., Cocks, A.C.F., Wu, C.Y., 2005. Experimental and numerical study of die filling, powder transfer and die compaction. Powder Metallurgy 48, 68-76.

Delleur, J.W., 2007. The Handbook of Groundwater Engineering. CRC Press.

Federzoni, L., Riedel, H., Coube, O., Oldenburg, M., Gethin, D.T., Mosbah, P., Virta, J., Martikainen, H., Frachon, A., Doremus, P., 1999. State of the art review: comparison of computer models representing powder compaction process. Powder Metallurgy 42, 301-311.

Ferguson, B.L., Krauss, T.M., 1990. Modeling ejection of die pressed parts. Advances in Powder Metallurgy 1, 147-164.

Hernández,J.A., 2009. Numerical modeling of crack formation in powder compaction based manufacturing problems. Technical University of Catalonia.

Hernández, J.A., Oliver, J., Cante, J.C., Weyler, R., 2011. Numerical modeling of crack formation in powder forming processes. International Journal of Solids and Structures 48, 292-316.

Modnet, P.M., 1999. Computer Modelling Group. Comparison of computer models representing powder compaction process. Powder Metallurgy 42, 301-311.
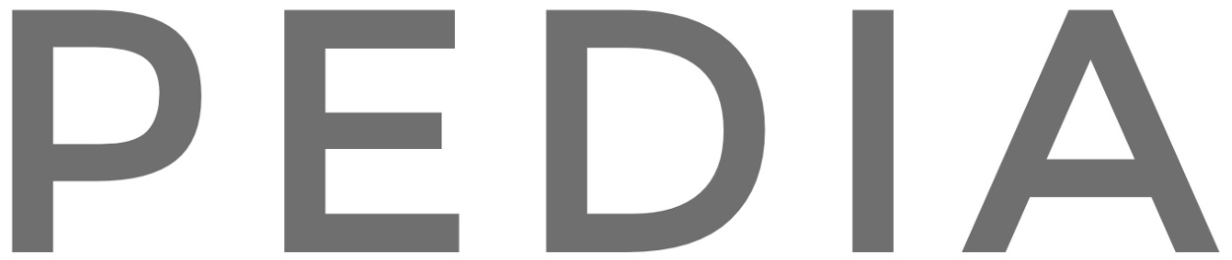